\title{
An alphavirus vector overcomes the presence of neutralizing antibodies and elevated numbers of Tregs to induce immune responses in humans with advanced cancer
}

\author{
Michael A. Morse,, ${ }^{1,2}$ Amy C. Hobeika, ${ }^{3}$ Takuya Osada, ${ }^{3}$ Peter Berglund, ${ }^{4}$ \\ Bolyn Hubby, ${ }^{5}$ Sarah Negri, ${ }^{4}$ Donna Niedzwiecki, ${ }^{2,6}$ Gayathri R. Devi, ${ }^{2,3}$ \\ Bruce K. Burnett, 2 Timothy M. Clay,2,3,7 Jonathan Smith, ${ }^{4}$ and H. Kim Lyerly,2,3,7
}

1Department of Medicine, ${ }^{2}$ Duke Comprehensive Cancer Center, and ${ }^{3}$ Department of Surgery, Duke University Medical Center, Durham, North Carolina, USA.

${ }^{4}$ AlphaVax Inc., Research Triangle Park, North Carolina, USA. ${ }^{5}$ Liquidia Technologies Inc., Research Triangle Park, North Carolina, USA.

${ }^{6}$ Department of Biostatistics and Bioinformatics and 7 Department of Immunology, Duke University Medical Center, Durham, North Carolina, USA.

\begin{abstract}
Therapeutic anticancer vaccines are designed to boost patients' immune responses to tumors. One approach is to use a viral vector to deliver antigen to in situ DCs, which then activate tumor-specific $T$ cell and antibody responses. However, vector-specific neutralizing antibodies and suppressive cell populations such as Tregs remain great challenges to the efficacy of this approach. We report here that an alphavirus vector, packaged in virus-like replicon particles (VRP) and capable of efficiently infecting DCs, could be repeatedly administered to patients with metastatic cancer expressing the tumor antigen carcinoembryonic antigen (CEA) and that it overcame high titers of neutralizing antibodies and elevated Treg levels to induce clinically relevant CEA-specific $T$ cell and antibody responses. The CEA-specific antibodies mediated antibody-dependent cellular cytotoxicity against tumor cells from human colorectal cancer metastases. In addition, patients with CEA-specific $T$ cell responses exhibited longer overall survival. These data suggest that VRP-based vectors can overcome the presence of neutralizing antibodies to break tolerance to self antigen and may be clinically useful for immunotherapy in the setting of tumor-induced immunosuppression.
\end{abstract}

\section{Introduction}

Efficacious therapeutic anticancer vaccines are now becoming a reality, but a number of challenges remain. More efficient platforms for delivering antigen to in situ DCs, critical for activation of $\mathrm{T}$ cell and antibody responses (1), are needed. Viral vectors are particularly promising for tumor antigen delivery because they may directly infect DCs and, by the nature of their engagement of innate immune signals, provide the appropriate cognate costimulation or "danger signals" that lead to enhanced DC activation breaking of tolerance through induction as well as expansion of the adaptive immune response. There has been extensive experience with numerous viral vectors based on poxvirus (vaccinia, fowlpox, canarypox), adenovirus, reovirus, herpesvirus, and others. Nonetheless, a challenge to the use of all viral vectors is the induction of neutralizing antibodies to proteins on the virus surface that precludes the repeated administration of recombinant vector-based vaccines, particularly those based on vaccinia and adenovirus $(2,3)$.

An attractive alternative is vaccination with recombinant alphavirus such as Venezuelan equine encephalitis (VEE) virus. The VEE structural protein genes may be replaced with a heterologous gene of interest to produce a recombinant RNA replicon capable of self replication and very high-level expression of the foreign gene, often reaching levels of $10 \%-15 \%$ of the total cell protein. These replicons may be packaged into virus-like replicon particles (VRP)

Conflict of interest: Peter Berglund and Sarah Negri are employed by AlphaVax Inc. H. Kim Lyerly is on the Scientific Advisory Board of AlphaVax Inc. Bolyn Hubby and Jonathan Smith were recently employed by AlphaVax Inc.

Citation for this article: J Clin Invest. 2010;120(9):3234-3241. doi:10.1172/JCI42672 by cotransfection of cells in vitro with helper RNAs encoding the viral structural proteins (4). VRP are morphologically identical to alphaviruses and efficiently express their foreign gene(s) at the site of inoculation, but the absence of the structural protein genes on the replicon prevents further amplification in the vaccine recipient. Other advantages of VRP are their tropism for, and maturation of (5), DCs, which could result in enhanced T cell activation. A number of preclinical studies have reported that VRP induce potent immunity despite the presence of neutralizing antibodies (4-12).

Recently, an alphavirus replicon vaccine for CMV was tested in CMV-seronegative volunteers. The vaccine was well tolerated and induced $\mathrm{T}$ cell and antibody responses against the highly immunogenic CMV proteins in these healthy young subjects (13). A critical question was whether we could immunize multiple times with the same vector against a self antigen if anti-VRP neutralizing antibodies were induced. We therefore tested to determine whether a VRP-based vector could be repeatedly administered to break tolerance to a nonimmunogenic self antigen in a clinically relevant setting, such as the immunomodulated environment of older patients with metastatic cancer.

We chose as the tumor antigen carcinoembryonic antigen (CEA) (14), widely expressed in colon, breast, lung, and other malignancies and well established as an immunotherapy target for cancer vaccines (15). We designed a VRP vaccine expressing CEA(6D), an Asn to Asp substitution in the CEA gene within the peptide capable of binding to HLA-A2, resulting in enhanced recognition by the T cell receptor (16). We tested its immunogenicity in murine models and demonstrated our ability to achieve improved immune response in preclinical models with a prime-and-boost strategy. We then gener- 
Table 1

Patient demographics

\begin{tabular}{|c|c|c|c|c|c|c|c|c|c|c|}
\hline $\begin{array}{l}\text { Patient } \\
\text { ID }\end{array}$ & Cohort & $\begin{array}{l}\text { Dose } \\
\text { (IU) } \times 10^{8}\end{array}$ & Diagnosis & Age & Sex & KPS & $\begin{array}{l}\text { Concomitant } \\
\text { therapy }\end{array}$ & $\begin{array}{c}\text { No. prior } \\
\text { chemo regimens }{ }^{A}\end{array}$ & $\begin{array}{l}\text { No. sites of } \\
\text { metastases }\end{array}$ & $\begin{array}{l}\text { Doses } \\
\text { received }\end{array}$ \\
\hline 0101 & 1 & 0.4 & Colon & 54 & M & 100 & No & 2 & 2 & 4 \\
\hline 0102 & 1 & 0.4 & Rectal & 49 & $\mathrm{~F}$ & 100 & No & 5 & 2 & 1 \\
\hline 0103 & 1 & 0.4 & Colon & 36 & $M$ & 100 & No & $4(1)$ & 2 & 4 \\
\hline 0105 & 1 & 0.4 & Colon & 65 & $\mathrm{~F}$ & 90 & Bevacizumab & 5 & 3 & 4 \\
\hline 0106 & 2 & 1 & Colon & 67 & $\mathrm{~F}$ & 90 & No & 4 & 1 & 4 \\
\hline 0107 & 2 & 1 & NSCLC & 53 & $\mathrm{~F}$ & 100 & No & 2 & 1 & 4 \\
\hline 0108 & 2 & 1 & NSCLC & 48 & M & 100 & No & 6 & 1 & 4 \\
\hline 0109 & 3 & 4 & Colon & 42 & $\mathrm{~F}$ & 100 & Bevacizumab & $5(1)$ & 1 & 4 \\
\hline 0110 & 3 & 4 & Colon & 42 & $M$ & 100 & No & 7 & 2 & 3 \\
\hline 0111 & 3 & 4 & Breast & 54 & $\mathrm{~F}$ & 100 & $\begin{array}{l}\text { Bevacizumab, } \\
\text { zoledronic acid }\end{array}$ & 11 & 1 & 4 \\
\hline 0112 & 3 & 4 & Colon & 59 & M & 90 & No & 4 & 0 & 8 \\
\hline 0113 & 3 & 4 & Colon & 53 & M & 100 & Bevacizumab & 4 & 1 & 4 \\
\hline 0114 & 3 & 4 & Pancreatic & 53 & $M$ & 90 & No & 2 & 1 & 6 \\
\hline 0116 & 3 & 4 & Colon & 52 & M & 100 & No & $3(1)$ & 1 & 4 \\
\hline 0117 & $\mathrm{Ph} \|$ & 4 & Rectal & 48 & M & 100 & No & 4 & 2 & 4 \\
\hline 0118 & $\mathrm{Ph} \|$ & 4 & Colon & 43 & M & 90 & Bevacizumab & 8 & 1 & 3 \\
\hline 0119 & $\mathrm{Ph} I \mathrm{I}$ & 4 & Rectal & 46 & $\mathrm{~F}$ & 90 & Bevacizumab & 4 & 1 & 5 \\
\hline 0120 & $\mathrm{Ph}$ II & 4 & Colon & 53 & $M$ & 100 & Bevacizumab & 3 & 2 & 4 \\
\hline 0121 & $\mathrm{Ph}$ II & 4 & Colon & 53 & $M$ & 90 & No & 5 & 2 & 4 \\
\hline 0122 & $\mathrm{Ph}$ II & 4 & Colon & 64 & $M$ & 80 & No & 4 & 1 & 2 \\
\hline 0123 & $\mathrm{Ph} \|$ & 4 & Colon & 69 & $M$ & 100 & Bevacizumab & 4 & 1 & 3 \\
\hline 0124 & $\mathrm{Ph}$ II & 4 & Colon & 64 & $M$ & 100 & No & 4 & 3 & 4 \\
\hline 0125 & $\mathrm{Ph}$ II & 4 & Colon & 60 & $M$ & 100 & No & 2 & 1 & 4 \\
\hline 0126 & $\mathrm{Ph}$ II & 4 & Colon & 40 & $\mathrm{~F}$ & 80 & Cetuximab & 4 & 5 & 4 \\
\hline 0127 & $\mathrm{Ph}$ II & 4 & Appendiceal & 55 & $M$ & 80 & No & 3 & 3 & 5 \\
\hline 0128 & $\mathrm{Ph} I \mathrm{I}$ & 4 & Colon & 62 & $\mathrm{~F}$ & 90 & No & 4 & 2 & 4 \\
\hline 0129 & $\mathrm{Ph} I I$ & 4 & Colon & 59 & $\mathrm{~F}$ & 100 & No & 2 & 2 & 2 \\
\hline 0130 & $\mathrm{Ph}$ II & 4 & Colon & 53 & $M$ & 90 & No & 2 & 0 & 4 \\
\hline
\end{tabular}

ANumbers in parentheses show prior CEA vaccine treatments. All patients had stage 4 cancer; those with 0 sites of metastases had undergone resection of their tumor or had no evidence of disease after chemotherapy. KPS, Karnofsky performance status; Ph., phase; NSCLC, non-small cell lung cancer.

ated GMP grade vector for this phase I/II clinical trial to evaluate the safety and clinical and immunologic efficacy of immunization with CEA(6D)-VRP (AVX701) in patients with advanced cancer.

The use of any cancer vaccine strategy must address the reality that there are number of immunoregulatory mechanisms, in particular, Tregs, invoked in the tumor microenvironment, regional lymph nodes, and peripheral blood that may limit or prevent the induction of clinically effective adaptive immune responses (17). CD4pos CD25 ${ }^{\text {hiFoxP3pos }}$ Tregs prevent uncontrolled proliferation of antigen-specific T cells (18). Elevated Treg levels can be detected in the peripheral blood, regional lymph nodes, and the tumor microenvironment of cancer patients compared with healthy individuals and are negatively associated with survival (19). Because Tregs appear to impair host immune responses against cancer (20), one approach to eliminating the influence of Tregs is by depleting them with the $\mathrm{CD} 25$-targeting immunotoxin denileukin diftitox prior to immunization (as others and we have done) $(21,22)$ or anti-CD25 antibody. However, these approaches could interfere with activated $\mathrm{T}$ cells that also express CD25. Viral vectors have been reported to provide persistent Toll-like receptor signals capable of reversing Treg-mediated CD8 tolerance in murine models (23). Therefore, we were interested in determining whether VRP-CEA(6D), would induce an adaptive immune response to self antigen in the setting of an immunosuppressive environment caused by elevated Tregs.
We performed a traditional " $3+3$ " phase I study of active immunotherapy with VRP-CEA(6D). We analyzed blood samples before each immunization and following completion of all immunizations for VRP-neutralizing antibodies, circulating Treg and CEA-specific adaptive humoral and cellular immune responses. Although we noted elevated levels of Tregs in the study patients and observed a rapid induction of anti-VRP neutralizing antibodies following the first immunization, we found that potent CEA-specific $T$ cell and antibody responses could nonetheless be induced by VRP-CEA(6D).

\section{Results}

Choice of multiple immunizations. We wished to develop an alphavirus-based vaccine in order to take advantage of the alphavirus' tropism for DCs and ability to generate large amounts of a protein of interest after infecting a cell. This was expected to cause infected DCs at the injection site to process and appropriately present CEA tumor antigen. Prior to initiating the clinical trial, we determined whether a single dose or a prime-plus-boost dose of VRP-CEA would achieve the highest levels of $T$ cell and antibody responses. C57BL/6-CEA-transgenic mice immunized twice with VRP-CEA showed a greater magnitude of CEA-specific $\mathrm{T}$ cell responses in an ELISpot assay (Supplemental Figure 1; supplemental material available online with this article; doi:10.1172/JCI42672DS1). In 

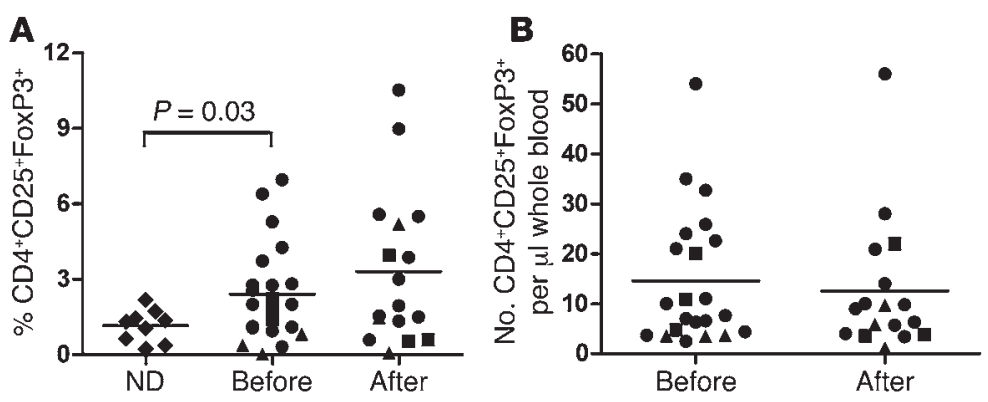

Figure 1

Treg analysis for patients and healthy volunteers. Patient blood was analyzed prevaccination and at week 12 ( 3 weeks following the fourth and final vaccination) by flow cytometry staining for $\mathrm{CD} 4{ }^{+} \mathrm{CD} 25^{+}$and intracellular FoxP3 to determine percentage of Tregs. (A) The percentage of CD4+CD25+FoxP3+ cells and the (B) number of $\mathrm{CD}^{+} \mathrm{CD} 25^{+} \mathrm{FoxP}^{+}$cells per $\mu \mathrm{l}$ whole blood are represented for each patient by a square for cohort 1, triangle for cohort 2, and circle for cohort 3 (maximal tolerated dose [MTD]) at prevaccination week 0 and postvaccination week 12. (A) The percentage of $\mathrm{CD} 4{ }^{+} \mathrm{CD} 25^{+} \mathrm{FoxP} 3^{+}$cells of normal donors (ND) was determined and analyzed using the same methods and is presented as a comparison (diamonds). The mean (bar) is also represented for ND, before vaccination, and after vaccination. Statistical significance is noted by Student's $t$ test between percentage of Tregs of normal donors and cancer patients in the study $(P=0.03)$. The differences in the percentage of Tregs before and after vaccination are not statistically significant for any of the vaccine doses $(P>0.2)$.

addition, therapeutic antitumor effects had been demonstrated in models following multiple vaccinations (data not shown). Furthermore, clinical trials targeting CEA have used multiple injections or priming followed by heterologous boosting. We therefore chose multiple immunizations for the clinical trial testing of this vector in patients with advanced cancer.

Patient demographics. Patients (18 male, 10 female, ages 36-69) who were diagnosed with colorectal $(n=23)$, appendiceal $(n=1)$, pancreas $(n=1)$, lung $(n=2)$, or breast $(n=1)$ cancer, had a performance status of $80 \%-100 \%$, and had failed a median of 4 prior chemotherapeutic regimens (range 2-11) were entered into the trial by cohorts and treated at 1 of 3 dose levels (Table 1 ). In the first cohort, 3 of 4 patients completed all 4 immunizations. One dropped out of the study due to development of a brain metastasis. In the second cohort, 3 of 3 patients completed all immunizations. In the third cohort, 6 of 7 patients completed all immunizations (with 1 dropping out after 3 immunizations due to progressive disease) and 2 continued receiving additional booster doses afterwards. Dose-limiting toxicity [DLT] was not reached, and the dose of $4 \times 10^{8} \mathrm{IU}$ was determined to be the maximal feasible dose. The trial allowed for 14 additional patients to be treated at the maximal feasible dose $\left(4 \times 10^{8} \mathrm{IU}\right)$. Of these next 14 patients, 10 received at least 4 immunizations, and 4 discontinued prior to the final immunization, all due to progression of disease. Two received an additional booster immunization. Overall, immunizations were well tolerated with 6 grade 3 events ( 1 each of alanine aminotransferase [ALT], bilirubin, neurological confusion, obstipation, dyspnea, and fatigue, all attributed to disease progression) and no grade 4 or higher events. The only injection site reactions observed were 2 grade 1 instances of injection site pain. There were no febrile reactions.

Elevated levels of Tregs in patients. We demonstrated that our gating strategy detecting $\mathrm{CD} 4{ }^{+} \mathrm{CD} 25^{+}$cells contained high levels of FoxP3 and could be sorted into a population that had immuno- suppressive effects in vitro (Supplemental Figure 2). We compared the circulating CD4Pos $C D 25^{\text {hi }}$ FoxP3pos Treg levels in the patients participating in this study with those of healthy volunteers. Consistent with previous reports (17), the percentage of Tregs was statistically greater $(P=0.03)$ for patients than healthy controls (Figure 1A). There was no statistically significant increase in Tregs following immunization both by percentage of $\mathrm{CD}^{+} \mathrm{T}$ cells and absolute number of CD4 ${ }^{\text {Pos }}$ CD2 $5^{\text {hi FoxP3 }}{ }^{\text {pos }}$ Tregs per microliter of blood (Figure 1, A and B).

Antigen-specific $T$ cell and antibody responses despite neutralizing antibody and elevated Treg levels. We measured the induction of anti-VRP neutralizing antibodies in patients in the high-dose cohort. We observed, after a single dose of VRP-CEA(6D), that the anti-VRP antibody titer increased dramatically and remained elevated throughout the immunizations (Figure 2A). Titers also remained elevated in the 4 patients in the high-dose cohort who continued to receive booster vaccinations every 3 months (with follow-up reaching 12 months) (data not shown). Because patients who did not receive the boosters went off study, we did not have longer term (>3 months) serum specimens available to determine whether the titer would remain elevated without the booster doses. However, because the first booster occurred at 3 months following the fourth injection, the data do suggest that the anti-VRP titers remain elevated at least 3 months following the first series of 4 injections.

The CEA-specific immune responses were analyzed at each time point by ELISpot and ELISA (Supplemental Figure 3 and Figure 2, B and C). We found a significant increase in immune response for at least 1 postvaccination time point versus prevaccination for all 3 assays in the high-dose cohort but not in the lower-dose cohorts (Supplemental Figure 3). Because we measured the immune response prior to each injection, we observed that the $\mathrm{T}$ cell response progressively increased beginning with the second or third immunization (Figure 2, B and C). For the 4 patients who received ongoing booster doses, the immune response levels remained at the levels induced by the initial 4 doses (data not shown). Again, we did not have long-term specimens for patients who received only the first 4 immunizations; however, because the first booster was at 3 months following the fourth injection, the data do suggest that the CEA-specific immune responses remain elevated at least 3 months following the first series of 4 injections. The data also suggest that further boosting does not continue to enhance the immune response, perhaps because the maximum attainable response has been reached.

We further characterized the ELISpot results using intracellular cytokine staining to identify the $\mathrm{CD}^{+}$(Figure 2D), and CD8 ${ }^{+} \mathrm{CEA}-$ specific $\mathrm{T}$ cell responses. The $\mathrm{CD} 4^{+} \mathrm{CEA}-$ specific $\mathrm{T}$ cell response increased by the final immunization in the majority $(9 / 13)$ of the patients. A CEA-specific CD8 ${ }^{+} \mathrm{T}$ cell response was also observed in $5 / 9$ patients analyzed in the high-dose cohort (data not shown). Taken together, these results illustrate that VRP-CEA(6D) induces both $\mathrm{CD}^{+}$and $\mathrm{CD} 8^{+} \mathrm{CEA}-$ specific $\mathrm{T}$ cell and antibody responses.

We then determined whether patients with low Tregs, defined as below the median of the Treg level for all patients in the study, had a different magnitude of immune response than those with high Tregs (i.e., above the median of the Treg levels for all patients). 
A
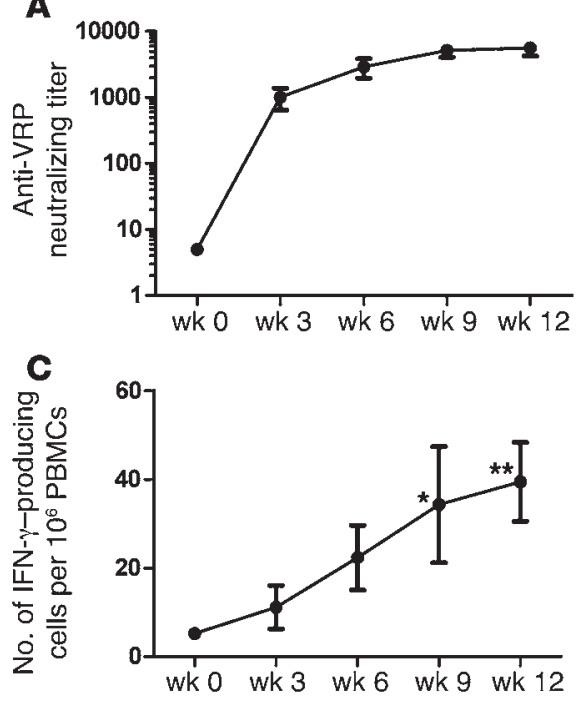
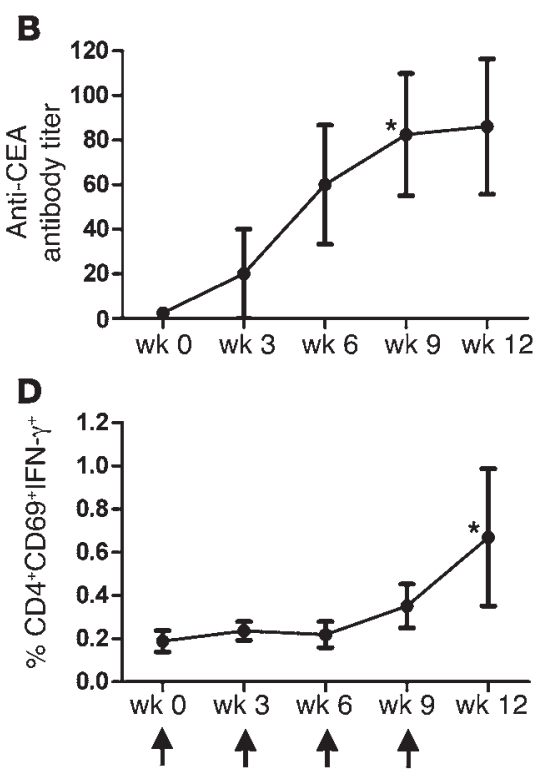

Figure 2

Immune analysis following VRP-CEA(6D) vaccination. Patient sera or PBMCs were analyzed before and after VRP-CEA(6D) for each immunization for MTD cohort by (A) anti-VRP microneutralization assay; (B) CEA ELISA; (C) VRP-CEA ELISpot; and (D) intracellular IFN- $\gamma$. (A) Patient sera were analyzed for anti-VRP antibodies by microneutralization assay for weeks $0,3,6,9$, and 12 . The antibody titer is presented for each week as mean \pm SD. The endpoint titer was defined as the last serum dilution at which there was at least $80 \%$ reduction in the number of GFP-positive cells compared with control wells. (B) Patient sera were tested against CEA protein by ELISA for weeks 0, 3, 6, 9, and 12. The antibody titer is represented over the course of the 4 vaccinations as mean \pm SEM. A single patient with an outlier titer of 1:1,600 at week 9 was not included in the data presented in the graph but was included for statistical analysis. ${ }^{*} P=0.02$. (C) Patient PBMCs were stimulated with VRPCEA (MOI 10) in an ELISpot assay. The number of IFN- $\gamma$-producing cells per $10^{6}$ PBMCs is presented as mean \pm SEM. ${ }^{*} P=0.01 ;{ }^{* *} P=0.005$. (D) Patient PBMCs were stimulated with VRP-CEA (MOI 1) and incubated for 12 hours in an intracellular cytokine assay. The percentage of cells that were $\mathrm{CD} 4^{+} \mathrm{CD} 69^{+} \mathrm{IFN}-\gamma^{+}$is presented as mean \pm SEM. Time of vaccinations is represented by arrows on the $x$ axis of graph (D). Regression analysis with repeated measures was used to analyze assay results over time. Time points where the mean significantly differed from baseline are indicated on the graph ( $P$ values are adjusted for multiple comparisons). ${ }^{*} P=0.01$.

The magnitude of the immune (CEA-specific T cell and antibody) responses was not statistically different regardless of whether patients had low- or high-peripheral blood Treg levels (Figure 3, A-C). Similarly, there was no significant correlation between the magnitude of anti-VRP neutralizing antibody titers and anti-CEA antibody titers or ELISpot levels (Figure 3, D and E). Hence, despite the induction of strong neutralizing antibodies and the presence of elevated Treg levels, the VRP-CEA(6D) remained capable of activating potent anti-CEA antibody and $\mathrm{T}$ cell responses.

Antitumor activity of vaccine-induced anti-CEA antibodies. We wished to determine the functional activity of vaccine-induced anti-CEA antibodies by analyzing whether antibodies in patient serum could bind a CEA-expressing colon cancer cell line and mediate antibody-dependent cellular cytotoxicity (ADCC) (Figure 4). CEA-specific antibodies in the sera of 2 patients from the highdose cohort bound to the $\mathrm{CEA}^{+}$cell line (SW1463) but not to the $\mathrm{CEA}^{-}$cell line (COLO320) at titers of 1:50 and 1:100 (Figure 4A). Furthermore, the CEA-specific antibody sera could mediate ADCC (Figure 4B). These data confirm that antibodies induced by VRP-CEA(6D) have functional capacity despite the presence of anti-VRP neutralizing antibodies and high Treg levels.
Clinical outcome. There were 2 patients with stable disease and 1 with a complete response (CR) of a small liver lesion (Figure 5A). Two patients had no evidence of disease prior to immunization and remained in that condition. Although numbers were small and there were not enough events to make a statistical comparison, nonetheless, there appeared to be better survival in the patients with an immune response by ELISpot (Figure 5B).

\section{Discussion}

The purpose of this study was to determine whether a vaccine based on recombinant alphaviruses could be repeatedly administered to break tolerance to a self antigen despite the expected elevated levels of immunosuppressive Tregs and the induction of neutralizing antibody against the VRP. Notwithstanding these challenges to vaccination, we hypothesized we would be able to break tolerance because preclinical studies had suggested higher magnitudes of $\mathrm{T}$ cell response after multiple immunizations with VRP-CEA compared with a single immunization (Supplemental Figure 1). Furthermore, in other preclinical studies (Supplemental Figure 4), we observed that even when VRP-encoding antigen was mixed with enough anti-VRP antibody to achieve neutralization of the VRP and then injected, there was still a high rate of $\mathrm{T}$ cell and antibody response against the antigen. Indeed, in the present clinical study, we observed that neutralizing antibodies developed after a single injection and peripheral blood Tregs were elevated in a substantial fraction of these advanced cancer patients, yet the VRP-CEA induced similarly high level CEAspecific $T$ cell and antibody responses with repeated doses of the same vector (homologous prime boost).

Although immunization against tumor antigens has been achieved with heterologous prime-boost strategies, homologous prime-boost strategies with vaccinia or adenovirus vectors have previously had limited success due to the induction of neutralizing antibodies that nullify the effect of successive immunizations $(2,3)$. The precise mechanism for retained functionality of VRPbased vaccines despite antivector neutralization is not known. However, a possible explanation could be the origin of the strain of VEE used for generating the glycoprotein coat of the VRP. The strain, named V3014, was generated following passages under a stringent selective pressure for accelerated penetration $(24,25)$ that resulted in multiple genetic alterations. It is possible that this rapidly cell-penetrating phenotype rendered the virion less sensitive to neutralization by virtue of being able to bind and infect cells in vivo before effective neutralization occurs. Another explanation, which does not exclude the first, could be the ability of 

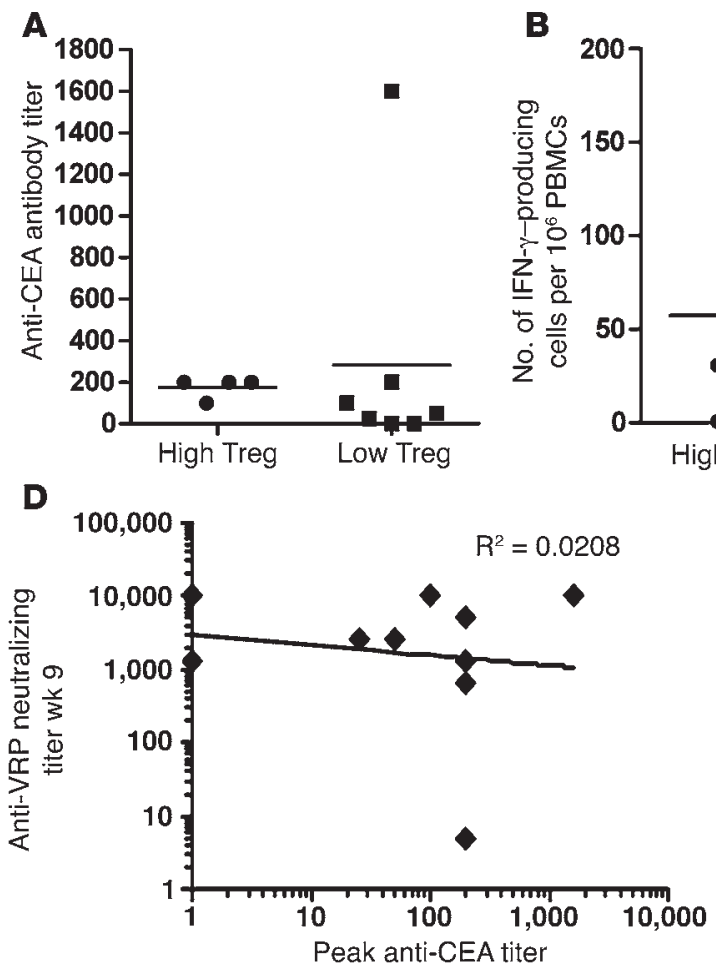
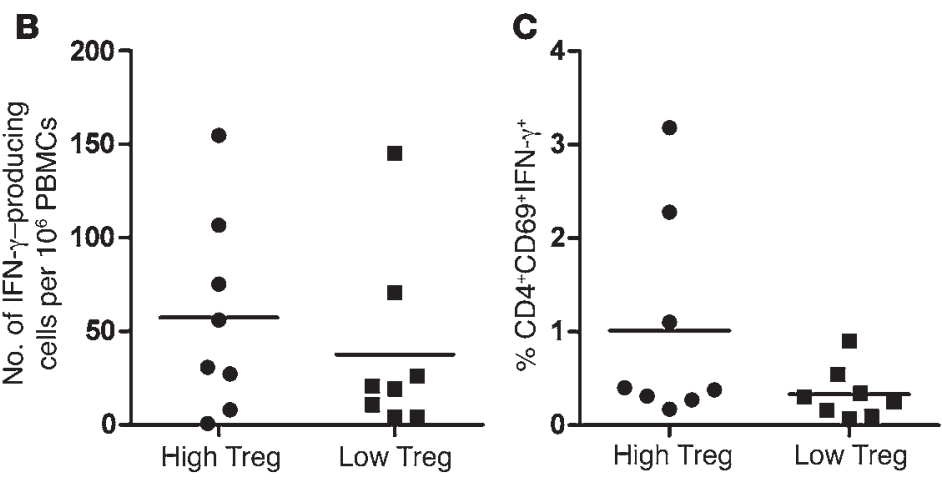

\section{E}

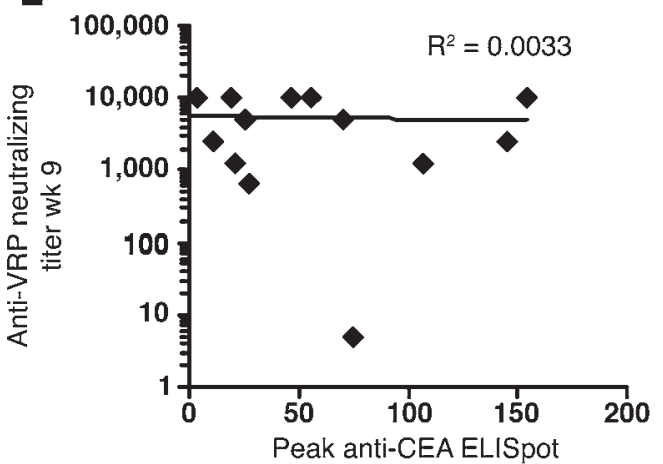

\section{Figure 3}

Comparison of the immune response in patients with high Treg levels and neutralizing antibody titers. (A-C) The immune responses from patients in the MTD cohort were split into 2 groups based on the absolute number of Tregs. Members of high-Treg and low-Treg subgroups were defined as patients that had greater or less than $10.4 \mathrm{CD} 4{ }^{+} \mathrm{CD} 25^{+} \mathrm{FoxP} 3+$ cells per $\mu$ whole blood as determined by FACS. This definition was based on the median number of $\mathrm{CD} 4{ }^{+} \mathrm{CD} 25^{+}$Foxp3 ${ }^{+}$per $\mu \mathrm{l}$ whole blood for patients in the study. The best response for each patient is represented by a circle (high Tregs) or square (low Tregs) along with the mean (bar) for (A) CEA ELISA; (B) VRP-CEA ELISpot; and (C) VRP-CEA intracellular IFN- $\gamma$. The difference in immune response between high-Treg and low-Treg subgroups was not statistically significant for any of the assays (Wilcoxon's rank sum; $P>0.05$ ). ( $\mathbf{D}$ and $\mathbf{E})$ Correlation between high-neutralizing antibody titers and (D) anti-CEA titer and (E) ELISpot response. The $R^{2}$ value represented on each graph shows no correlation for anti-VRP titers versus (D) anti-CEA antibodies and versus (E) CEA ELISpot.

VRP to target cells efficiently in vivo even when coated with otherwise neutralizing antibodies. Another explanation could be the fact that VRP is able to infect and replicate its genome in DCs, which are capable of taking up exogenous particular material such as opsonized virus. Finally, because VRP generate large amounts of the encoded protein (CEA), even a small number of VRP reaching DCs without neutralizing antibody could be sufficient to induce potent immune responses.

There are several possible explanations for why we observed similar magnitudes and rates of CEA-specific $\mathrm{T}$ cell and antibody responses regardless of the level of Tregs. Viral vectors have been reported to provide persistent Toll-like receptor signals capable of reversing Treg-mediated CD8 tolerance in murine models (23). Of course, it is possible that the Foxp $3^{+}$cells are not functional Tregs or that the actual anatomic location for breaking Treg-mediated tolerance to antibody and $\mathrm{T}$ cell response is not blood. However, we have previously observed that blood Tregs are functional (Supplemental Figure 2) and depleting them from peripheral blood was associated with enhanced $\mathrm{T}$ cell responses (21). Therefore, we believe that the elevated Tregs observed in the peripheral blood of the patients in the current study would have the capability of inhibiting $\mathrm{T}$ cell responses, yet the alphavirus vaccine was able to evade this effect. There is less published on the effect of Tregs on antibody responses, however.
Fields reported that the secretion of antibodies by anti-chromatin $\mathrm{B}$ cells is blocked by the injection of $\mathrm{CD} 4^{+} \mathrm{CD} 25^{+}$Treg cells (26). This suggests that Tregs can have a negative effect on antibody responses as well.

Although the rate of clinical responses was low in this heavily pretreated population, we did observe a regression of a liver metastasis in a patient with pancreatic cancer; 2 patients had stable disease, and 2 patients with no evidence of disease remained in remission. All of these instances of clinical benefit were in the high-dose cohort, suggesting the possibility of a dose-clinical response relationship. It is possible we would see greater clinical benefit with higher doses of VRP-CEA. However, for what we believe is the first-in-human clinical study, we limited the dose to $4 \times 10^{8} \mathrm{IU}$, which was the highest level for which we had preclinical toxicology data available.

In summary, we were able to treat advanced cancer patients with repeated immunizations with a recombinant alphaviral vector and to break tolerance to nonmutated CEA despite the induction of neutralizing antibody against the VRP and elevated Treg levels. This is the first study we are aware of that shows a viral vector overcoming neutralizing antibodies and elevated Tregs in cancer patients. Future studies will focus on enhancing the immune response further by the addition of cytokines such as IL-12 and studying the VRP in patients with less advanced disease. 
A

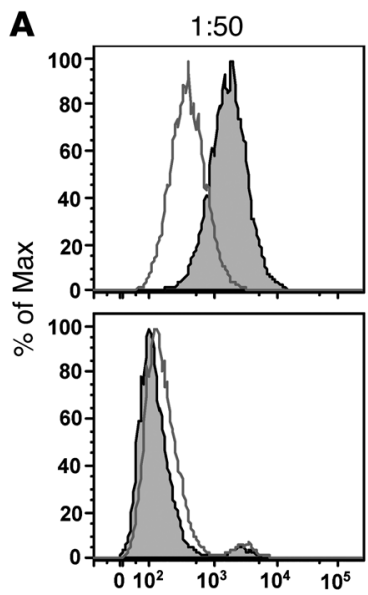

$1: 100$

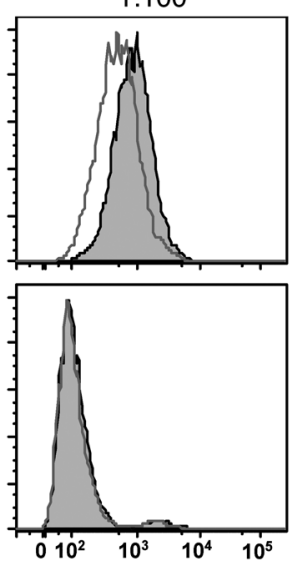

$1: 200$

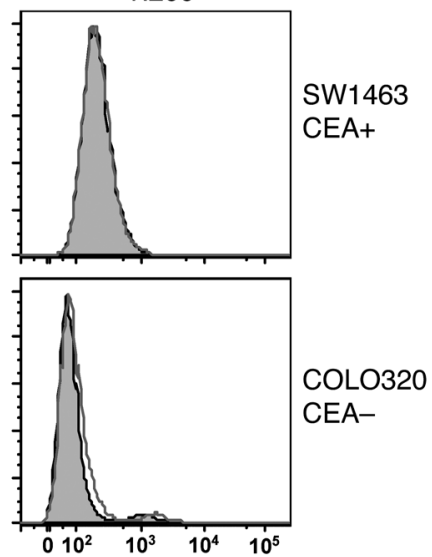

B

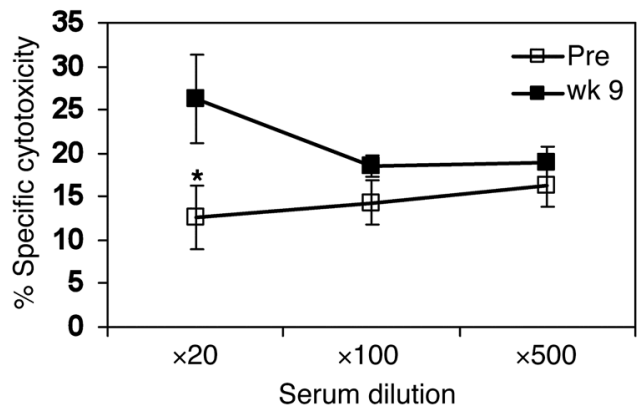

\section{Figure 4}

Serum cell binding and ADCC assay. (A) Sera from high-dose cohort patient AVX701-0121 were incubated with CEA-expressing colon tumor cell line SW1463 (CEA ${ }^{+}$and non-CEAexpressing colon tumor line COLO320 (CEA $\left.{ }^{-}\right)$. Results are represented as histograms of MFI for 1:50, 1:100, and 1:200 dilutions. Binding of prevaccination serum antibodies is represented by white peaks, and the postvaccination serum is represented by the gray peaks. The top row of histograms binds against SW1463 $\left(\mathrm{CEA}^{+}\right)$cell line and the lower row COLO320 cell line. The ELISA titer for patient AVX7010121 was 1:200. (B) Serial dilutions of pre- and postimmunization sera from high-dose cohort patient AVX701-0109 were incubated with CEA-expressing colon tumor cell line SW1463 $\left(C E A^{+}\right)$in an ADCC assay. The percentage of specific lysis is presented as mean \pm SD. ${ }^{*} P<0.01$ for prevaccination versus week 9 serum at 1:20 ratio. The ELISA titer for patient AVX701-0109 was 1:1,600.

\section{Methods}

Generation of CEA(6D) VRP. We generated a replicon expression plasmid containing the CEA gene with the Cap-1(6D) mutation (15) called CEA(6D) VRP (AVX701) (Supplemental Figure 5) as previously described $(9,13,27)$. It was formulated so that the maximum feasible dose to administer in the clinical trial was $4 \times 10^{8} \mathrm{IU}$.

Preclinical studies to assess immunogenicity in mice. Human-CEA transgenic mice (28) were vaccinated via bilateral footpad injection on day 0 with VRP-CEA $\left(1 \times 10^{7}\right.$ virus particles $)$ and day 21 with either saline or VRPCEA. Fourteen days later, mice were euthanized and splenocytes were collected for analysis. IFN- $\gamma$ ELISpot assay was performed to examine the CEA-specific T cell responses (4 mice per condition) to an overlapping polypeptide mix for human CEA. All animal studies were approved by the Duke IACUC.
Protocol schema and patient treatment. The study was performed under an FDA-approved Investigational New Drug Exemption (IND) and registered at ClinicalTrials.gov (NCT00529984). Participants were recruited from oncology clinics at Duke University Medical Center and provided signed informed consent approved by the Duke Institutional Review Board. Eligibility requirements included metastatic cancer expressing CEA and adequate hematologic, renal, and hepatic function. Trial participants were required to have received treatment with standard therapy known to have a possible overall survival benefit or refused such therapy. Exclusion criteria included chemotherapy or radiation within the prior 4 weeks, history of autoimmune disease, viral hepatitis, HIV, or use of immunosuppressives. Some patients continued bevacizumab or cetuximab. Prior CEA immunotherapy was permitted.

This study used a standard $3+3$ dose escalation strategy with DLTs defined as grade 3 or 4 major organ toxicity. The CEA(6D) VRP injections were given

\section{A}

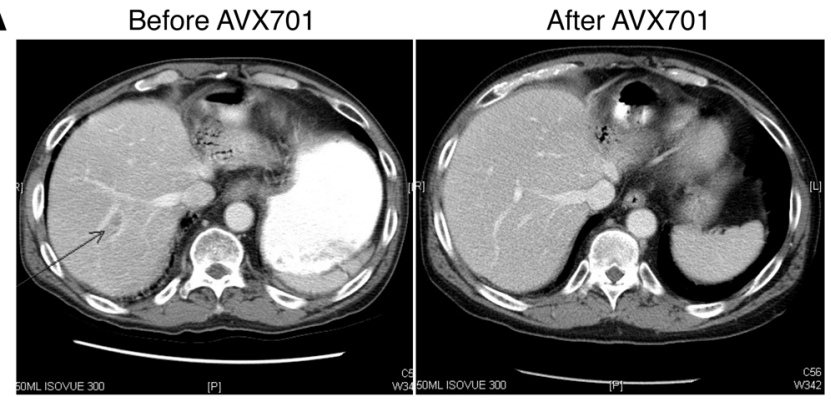

B

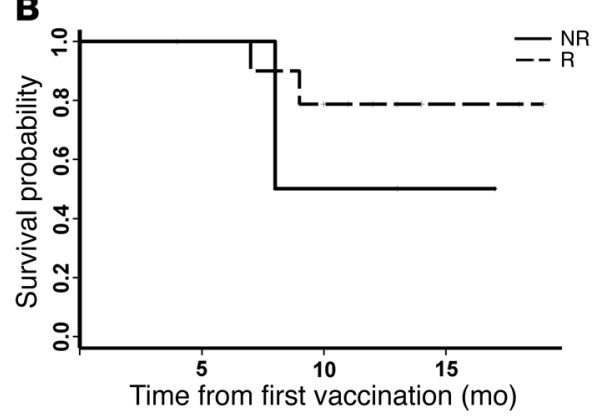

Figure 5

Clinical response. (A) CT scan from a pancreatic cancer patient refractory to chemotherapy and radiotherapy. Arrow indicates a liver metastasis that resolved after vaccination. (B) Kaplan-Meier estimates of overall survival by best immune response (by ELISpot) for patients treated on VRP-CEA(6D) (AVX701) at MTD $\left(4 \times 10^{8} \mathrm{IU}\right)$. 
into the deltoid muscle and alternated side-to-side at each visit. The doses were as follows: cohort 1: CEA(6D) VRP at a dose of $4 \times 10^{7} \mathrm{IU}$ in $0.5 \mathrm{ml}$ i.m. every 3 weeks for 4 immunizations.; cohort 2: CEA(6D) VRP at a dose of $1 \times 10^{8}$ IU in $0.125 \mathrm{ml}$ i.m. every 3 weeks for 4 immunizations; cohort 3: CEA(6D) VRP at a dose of $4 \times 10^{8} \mathrm{IU}$ in $0.5 \mathrm{ml}$ i.m. every 3 weeks for 4 immunizations. Higher doses were not possible due to limits on the amount of clinical-grade study drug. Following establishment of the safety of the dose of $4 \times 10^{8} \mathrm{IU}$, an additional 14 patients received CEA(6D) VRP at this dose. Patients with stable disease after the 4 study injections were permitted to receive booster doses of CEA(6D) VRP every 3 months until progressive disease.

Enumeration of Tregs. Whole blood from before and after immunizations was stained using anti-CD25, anti-CD4, anti-CD3, and anti-CD45 with BD Bioscience TruCount tubes as previously described (ref. 21 and Supplemental Figure 3). $\mathrm{CD} 4{ }^{+} \mathrm{CD} 25^{+} \mathrm{FoxP}^{+} \mathrm{T}$ cells were identified by gating on the $\mathrm{CD} 4{ }^{\mathrm{Pos}} \mathrm{CD} 25^{\text {hi }}$ cells, and the percentage of $\mathrm{FoxP}^{+}$cells and the number of $\mathrm{CD} 4^{+} \mathrm{CD} 25^{+} \mathrm{FoxP} 3^{+}$cells per microliter of blood determined. Peripheral blood was obtained from healthy donors who signed informed consent forms approved by the Duke Institutional Review Board.

Analysis of CEA-specific T cell responses by IFN- $\gamma$ ELISpot assay and intracellular cytokine staining. For immunologic analyses, freshly isolated PBMCs from peripheral blood were collected at weeks $0,3,6,9$, and 12 and analyzed for antigen-specific reactivity by ELISpot and intracellular cytokine assays as previously described (21).

Analysis of anti-CEA antibodies by ELISA. Patient sera were collected at weeks $0,3,6$, 9, and 12. 96-well plates were coated with whole CEA protein (100 ng/well) and incubated with $100 \mu \mathrm{l}$ of serum serially diluted $1: 10$ to $1: 31,250$. Titers were defined as the highest dilution such that the mean absorbance was equal to twice the negative control.

Analysis of anti-vector responses with a VRP microneutralization assay. To determine anti-vector responses, antibodies to VRP were measured using a microneutralization assay as previously described (13). VRP expressing GFP was mixed with serial dilutions of patient sera and then added to Vero cells. The number of cells expressing GFP in each well was determined by fluorescence microscopy.

Analysis of anti-CEA antibody binding to $C E A^{+}$buman colon cancer cells by FACS. We have adapted a methodology reported by Wei et al. to measure vaccine-induced antibodies in serum by flow cytometry (29). Serial dilutions of sera were incubated with CEA-expressing colon tumor cell line SW1463 $\left(\mathrm{CEA}^{+}\right)$and non-CEA-expressing colon tumor line COLO320 $\left(\mathrm{CEA}^{-}\right)\left(3 \times 10^{5}\right.$ cells $)$ for 1 hour at $4^{\circ} \mathrm{C}$ and analyzed for antibody binding to cells by FACS on a BD LSRII.

$A D C C$ assay. Patient PBMCs were incubated in rhIL-2 (1000 IU/ml) overnight. SW1463 $\left(\mathrm{CEA}^{+}\right)$and Colo320 (CEA $)$colorectal cancer cell lines were labeled with ${ }^{51} \mathrm{chromium}$ and placed into 96 -well V-bottomed plates for use as target cells. Patient sera were added at 1:20, 1:100, and 1:500 dilutions and further incubated for 1 hour. PBMCs were then added to the wells to achieve a 100:1 effector/target ratio. ${ }^{51}$ Chromium released into the supernatant was analyzed by MicroBeta Plus Scintillation counter (Wallac). Cytotoxicity was calculated as percentage of target cell lysis $=100 \times(\mathrm{cpm}$ of experimental release $-\mathrm{cpm}$ of spontaneous release)/(cpm of maximum release - cpm of spontaneous release).
Analysis of clinical activity. Clinical activity was assessed by applying the RECIST criteria to CT or MRI scans obtained before and after all immunizations. Peripheral blood tumor CEA level was assessed if available. Patients were followed long-term for survival.

Statistics. A positive immune response by ELISpot was defined as described at the 2002 Society of Biologic Therapy Workshop on "Immunologic Monitoring of Cancer Vaccine Therapy": a T cell response is considered positive if the mean number of spots in 6 wells with antigen exceeds the mean number of spots in 6 control wells by 10 and the difference between the mean of the 6 wells containing antigen and the 6 control wells is statistically significant at a level of $P<0.05$ using the Student's $t$ test (30). A positive response in the intracellular cytokine assay was defined as the percentage of positive cells by FACS analysis after vaccination greater or equal to twice the prevaccination sample. A positive result in the ELISA was defined as a 1:25 antibody titer. Titer is defined as twice the absorbance of the negative control serum.

Wilcoxon's signed rank test was used to compare paired measurements of immune response and Wilcoxon's rank sum test was used to compare measurements of immune response between 2 independent groups. The relationship between immune response outcome and time (week) was investigated using regression analysis with week considered as a continuous variable. Linear, quadratic, and cubic terms were included in the model. Repeated measures within patients were modeled by compound symmetry. The data were also analyzed considering week as a categorical variable. Under this model, multiple comparisons were conducted for each week versus week 0 , adjusting the level of significance using Dunnett's method.

\section{Acknowledgments}

The authors wish to thank Delila Serra, Amanda Bradshaw, Karrie Comatas, and Wiguins Etienne for their performance of the immune monitoring assays; Liz Anderson and Nancy J. Lee for their nursing management of the patients on the clinical trial; Elizabeth Reap for immunogenicity studies with neutralized VRP; and Whitney Lewis for the molecular construction of VRP-CEA. This work was supported by a grant from the NIH National Cancer Institute (principal investigator: H.K. Lyerly [NCI PO1CA078673]) and supported in part by the Department of Defense (PI: G.R. Devi [W81XWH-07-1-0392]).

Received for publication February 15, 2010, and accepted in revised form June 16, 2010.

Address correspondence to: Michael A. Morse, Rm. 403 MSRB 1 Box 3233, Duke University Medical Center, Durham, North Carolina 27710, USA. Phone: 919.681.3480; Fax: 919.681.7970; E-mail: morse004@mc.duke.edu.

Jonathan Smith's present address is: Liquidia Technologies, Research Triangle Park, North Carolina, USA.
1. Banchereau J, Klechevsky E, Schmitt N, Morita R, Palucka K, Ueno H. Harnessing human dendritic cell subsets to design novel vaccines. Ann N Y Acad Sci. 2009;1174:24-32.

2. Kundig TM, Kalberer CP, Hengartner CP, Zinkemagel RM. Vaccination with two different vaccinia recombinant viruses: long-term inhibition of secondary vaccination. Vaccine. 1993;11(11):1154-1158.

3. Sumida SM, et al. Neutralizing antibodies to adenovirus serotype 5 vaccine vectors are directed primarily against the adenovirus hexon protein. J Immunol. 2005;174(11):7179-7185.
4. Pushko P, Parker M, Ludwig GV, Davis NL, Johnston RE, Smith JF. Replicon-helper systems from attenuated Venezuelan equine encephalitis virus: expression of heterologous genes in vitro and immunization against heterologous pathogens in vivo. Virology. 1997;239(2):389-401.

5. Moran TP, et al. Alphaviral vector-transduced dendritic cells are successful therapeutic vaccines against neu-overexpressing tumors in wild-type mice. Vaccine. 2007;25(36):6604-6612.

6. Rayner JO, Dryga SA, Kamrud KI. Alphavirus vectors and vaccination. Rev Med Virol.
2002;12(5):279-296

7. Cassetti MC, et al. Antitumor efficacy of Venezuelan equine encephalitis virus replicon particles encoding mutated HPV16 E6 and E7 genes. Vaccine. 2004;22(3-4):520-527.

8. Durso RJ, et al. A novel alphavirus vaccine encoding prostate-specific membrane antigen elicits potent cellular and humoral immune responses. Clin Cancer Res. 2007;13(13):3999-4008.

9. Goldberg SM, et al. Comparison of two cancer vaccines targeting tyrosinase: plasmid DNA and recombinant alphavirus replicon particles. Clin 
Cancer Res. 2005;11(22):8114-8121.

10. Hubby B, et al. Development and preclinical evaluation of an alphavirus replicon vaccine for influenza. Vaccine. 2007;25(48):8180-8189.

11. Nelson EL, et al. Venezuelan equine encephalitis replicon immunization overcomes intrinsic tolerance and elicits effective anti-tumor immunity to the 'self' tumor-associated antigen, neu in a rat mammary tumor model. Breast Cancer Res Treat. 2003;82(3):169-183.

12. Wang X, Wang JP, Maughan MF, Lachman LB. Alphavirus replicon particles containing the gene for HER2/neu inhibit breast cancer growth and tumorigenesis. Breast Cancer Res. 2005;7(1):R145-R155.

13. Bernstein DI, etal. Randomized, double-blind, Phase 1 trial of an alphavirus replicon vaccine for cytomegalovirus in CMV seronegative adult volunteers. Vaccine. 2009;28(2):484-493.

14. Hammarström $S$. The carcinoembryonic antigen (CEA) family: structures, suggested functions and expression in normal and malignant tissues. Semin Cancer Biol. 1999;9(2):67-81.

15. Berinstein NL. Carcinoembryonic antigen as a target for therapeutic anticancer vaccines: a review. J Clin Oncol. 2002;20(8):2197-2207.

16. Zaremba S, Barzaga E, Zhu M, Soares N, Tsang KY, Schlom J. Identification of an enhancer agonist cytotoxic $\mathrm{T}$ lymphocyte peptide from human carcinoembryonic antigen. Cancer Res. 1997;57(20):4570-4577.

17. Beyer M, Schultze JL. Regulatory T cells in cancer. Blood. 2006;108(3):804-811.

18. Piccirillo CA, Shevach EM. Naturally-occurring CD4+CD25+ immunoregulatory T cells: central players in the arena of peripheral tolerance. Semin Immunol. 2004;16(2):81-88.

19. Curiel TJ, et al. Specific recruitment of regulatory $\mathrm{T}$ cells in ovarian carcinoma fosters immune privilege and predicts reduced survival. Nat Med. 2004;10(9):942-949.

20. Ahmad M, Rees RC, Ali SA. Escape from immunotherapy: possible mechanisms that influence tumor regression/progression. Cancer Immunol Immunother. 2004;53(10):844-854.

21. Morse MA, et al. Depletion of human regulatory $T$ cells specifically enhances antigen-specific immune responses to cancer vaccines. Blood. 2008;112(3):610-618.

22. Dannull J, et al. Enhancement of vaccine-mediated antitumor immunity in cancer patients after depletion of regulatory $\mathrm{T}$ cells. $J$ Clin Invest. 2005;115(12):3623-3633.

23. Yang Y, Huang CT, Huang X, Pardoll DM. Persistent Toll-like receptor signals are required for reversal of regulatory $\mathrm{T}$ cell-mediated CD8 tolerance. Nat Immunol. 2004;5(5):508-515.
24. Davis NL, et al. Attenuating mutations in the E2 glycoprotein gene of Venezuelan equine encephalitis virus: construction of single and multiple mutants in a full-length cDNA clone. Virology. 1991;183(1):20-31.

25. Johnston RE, Smith JF. Selection for accelerated penetration in cell culture coselects for attenuated mutants of Venezuelan equine encephalitis virus. Virology. 1988;162(2):437-443.

26. Fields ML, et al. CD4+CD25+ regulatory $\mathrm{T}$ cells inhibit the maturation but not the initiation of an autoantibody response. J Immunol. 2005;175(7):4255-4264.

27. Talarico T, Maughan M, Pancorbo B, Ruiz J, Graham A. Development and manufacture of alphavaccines. Bioprocessing J. 2006;5(3):8-14.

28. Eades-Perner AM, et al. Mice transgenic for the human carcinoembryonic antigen gene maintain its spatiotemporal expression pattern Cancer Res. 1994;54(15):4169-4176.

29. Piechocki MP, Pilon SA, Wei WZ. Quantitative measurement of anti-ErbB-2 antibody by flow cytometry and ELISA. J Immunol Methods. 2002;259(1-2):33-42.

30. Keilholz U, et al. Immunologic monitoring of cancer vaccine therapy: results of a workshop sponsored by the Society for Biological Therapy. J Immunother. 2002;25(2):97-138. 